\title{
EXPONENTIAL DICHOTOMIES \\ FOR ALMOST PERIODIC EQUATIONS
}

\author{
KENNETH J. PALMER
}

\begin{abstract}
It is shown that if an almost periodic equation has an exponential
\end{abstract} dichotomy on a sufficiently long finite interval, then it has one on $(-\infty, \infty)$.

1. Introduction. It is known (Coppel [2, p. 70]) that if an almost periodic equation

$$
\dot{x}=A(t) x
$$

has an exponential dichotomy on an interval $[a, \infty)$, then it has one on $(-\infty, \infty)$. The main purpose of this paper is to show that if equation (1) has an exponential dichotomy on some sufficiently long finite interval, then it has one on $(-\infty, \infty)$.

2. The main result. Our main theorem is closely related to Theorem 1 in Palmer [4]. However, because of the almost periodicity, the conclusions are stronger. For the proof of the theorem, we need a lemma.

LEMMA 1. Let $A(t)$ be an $n \times n$ matrix function, defined and continuous on an interval $[a, b]$. Write $M=\sup _{a \leqslant t \leqslant b}|A(t)|$. Let $B(t)$ be another $n \times n$ matrix function, defined and continuous on $[a, b]$ also, such that $|B(t)-A(t)|<\delta$ for $a \leqslant t \leqslant b$. Then if $X(t)$ is a fundamental matrix for equation (1) and $Y(t)$ is one for the equation

$$
\dot{y}=B(t) y,
$$

the inequality

$$
\left|Y(t) Y^{-1}(u)-X(t) X^{-1}(u)\right| \leqslant e^{M(b-a)}\left[e^{\delta(b-a)}-1\right]
$$

holds for $a \leqslant t, u \leqslant b$.

Proof. First note that it follows from Gronwall's lemma (cf. Coppel [1, p. 19]) that

$$
\left|X(t) X^{-1}(u)\right| \leqslant e^{M|t-u|}
$$

for $a \leqslant t, u \leqslant b$.

Now $W(t)=Y(t) Y^{-1}(u)-X(t) X^{-1}(u)$ is the solution of the inhomogeneous equation

$$
\dot{W}=A(t) W+[B(t)-A(t)]\left[W(t)+X(t) X^{-1}(u)\right]
$$

Received by the editors July 23, 1986.

1980 Mathematics Subject Classification (1985 Revision). Primary 34A30, 34C27; Secondary 34D05, $58 \mathrm{~F} 15$.

Key words and phrases. Linear system, almost periodic, exponential dichotomy. 
such that $W(u)=0$ and so by the variation of constants formula,

$$
W(t)=\int_{u}^{t} X(t) X^{-1}(\sigma)[B(\sigma)-A(\sigma)]\left[W(\sigma)+X(\sigma) X^{-1}(u)\right] d \sigma .
$$

Hence if $a \leqslant u \leqslant t \leqslant b$,

$$
\begin{aligned}
|W(t)| & \leqslant \delta \int_{u}^{t} e^{M(t-\sigma)}\left[|W(\sigma)|+e^{M(\sigma-u)}\right] d \sigma \\
& =\delta(t-u) e^{M(t-u)}+\delta \int_{u}^{t} e^{M(t-\sigma)}|W(\sigma)| d \sigma .
\end{aligned}
$$

Set $v(t)=e^{-M t}|W(t)|$. Then for $a \leqslant u \leqslant t \leqslant b$,

$$
v(t) \leqslant \delta(t-u) e^{-M u}+\delta \int_{u}^{t} v(\sigma) d \sigma .
$$

Then Gronwall's lemma implies that

$$
v(t) \leqslant \delta(t-u) e^{-M u}+\delta^{2} e^{-M u} \int_{u}^{t}(\sigma-u) e^{\delta(t-\sigma)} d \sigma .
$$

Integrating by parts, we get $v(t) \leqslant e^{-M u}\left\{e^{\delta(t-u)}-1\right\}$. Hence for $a \leqslant u \leqslant t \leqslant b$,

$$
\left|Y(t) Y^{-1}(u)-X(t) X^{-1}(u)\right| \leqslant e^{M|t-u|}\left\{e^{\delta|t-u|}-1\right\} .
$$

A similar inequality can be proved when $a \leqslant t \leqslant u \leqslant b$ and so when $a \leqslant t, u \leqslant b$

$$
\left|Y(t) Y^{-1}(u)-X(t) X^{-1}(u)\right| \leqslant e^{M(b-a)}\left\{e^{\delta(b-a)}-1\right\} .
$$

This completes the proof of the lemma.

Now we introduce some notation and define what is meant by an exponential dichotomy. Let $A(t)$ be an $n \times n$ matrix function, defined and continuous on an interval $J$. Here, and in the sequel, we denote by $X(t)$ a fundamental matrix for equation (1). Equation (1) is said to have an exponential dichotomy on $J$ with constants $K \geqslant 1, \alpha>0$, and projection $P$ if

$$
\begin{array}{ll}
\left|X(t) P X^{-1}(u)\right| \leqslant K e^{-\alpha(t-u)} & (u \leqslant t ; t, u \in J), \\
\left|X(t)(I-P) X^{-1}(u)\right| \leqslant K e^{-\alpha(u-t)} & (u \geqslant t ; t, u \in J) .
\end{array}
$$

The function $P(t)=X(t) P X^{-1}(t)$ is called the projection matrix function associated with the dichotomy.

If $\delta$ is a positive number, a real number $\tau$ is said to be a $\delta$-translation number for $A(t)$ if

$$
|A(t+\tau)-A(t)|<\delta
$$

for all real $t$. If for every $\delta>0$ the set of $\delta$-translation numbers is relatively dense on the real line, $A(t)$ is said to be almost periodic.

THEOREM 1. Let $A(t)$ be an $n \times n$ matrix function, defined and almost periodic on $(-\infty, \infty)$, such that equation (1) has an exponential dichotomy on an interval $[a, a+T]$ with constants $K, \alpha$.

Let $\delta$ be the largest positive number such that $e^{M h}\left(e^{\delta h}-1\right) \leqslant 1$, where $M=$ $\sup |A(t)|$ and $h=\alpha^{-1}\left(\sinh ^{-1} 4+\log K\right)$. Then if $T>0$ is so large that $T \geqslant 4 h$ and every interval of length $T / 2$ contains a $\delta$-translation number for $A(t)$, equation (1) has an exponential dichotomy on $(-\infty, \infty)$ with constants $L$ and $\beta=h^{-1} \log 3$, where $L$ depends only on $M, K$, and $\alpha$. 
Proof. Let $s$ be a given real number. Then the interval $\left[s-\frac{3}{4} T-a, s-\frac{1}{4} T-a\right]$ contains a $\delta$-translation number $\tau$ for $A(t)$. So for all $t$

$$
|A(t)-A(t-\tau)|<\delta \text {. }
$$

Now since $\left[s-\tau-\frac{1}{4} T, s-\tau+\frac{1}{4} T\right] \subset[a, a+T]$, the equation

$$
\dot{y}=A(t-\tau) y
$$

has an exponential dichotomy on $\left[s-\frac{1}{4} T, s+\frac{1}{4} T\right]$ with constants $K, \alpha$. Then since $T \geqslant 4 h$ and

$$
K^{-1} e^{\alpha h}-K e^{-\alpha h}=2 \sinh (\alpha h-\log K)=8,
$$

it follows from Coppel [2, p. 14] that if $y(t)$ is any solution of equation (4),

$$
|y(s)| \leqslant \frac{1}{4} \sup \{|y(t)|:|t-s| \leqslant h\} \text {. }
$$

Now let $x(t)$ be any solution of equation (1) and let $y(t)$ be the solution of equation (4) with $y(s)=x(s)$. It follows from (3), Lemma 1, and the definition of $\delta$ that for $|t-s| \leqslant h$,

$$
|y(t)-x(t)|=\left|\left[X(t-\tau) X^{-1}(s-\tau)-X(t) X^{-1}(s)\right] x(s)\right| \leqslant|x(s)| .
$$

So

$$
\begin{aligned}
|x(s)| & =|y(s)| \leqslant \frac{1}{4} \sup \{|y(t)|:|t-s| \leqslant h\} \\
& \leqslant \frac{1}{4} \sup \{|x(t)|+|y(t)-x(t)|:|t-s| \leqslant h\} \\
& \leqslant \frac{1}{4} \sup \{|x(t)|:|t-s| \leqslant h\}+\frac{1}{4}|x(s)| .
\end{aligned}
$$

Hence for all solutions $x(t)$ of equation (1) and all real $s$,

$$
|x(s)| \leqslant \frac{1}{3} \sup \{|x(t)|:|t-s| \leqslant h\} .
$$

Also, from Gronwall's lemma, $|x(t)| \leqslant e^{M|t-s|}|x(s)|$ for all $t, s$.

Then it follows from Proposition 1 in Coppel [2, p. 14] and its proof that if $x(t)$ is a solution of (1) bounded on $[0, \infty)$

$$
|x(t)| \leqslant N e^{-\beta(t-u)}|x(u)| \quad(t \geqslant u)
$$

and if $x(t)$ is a solution bounded on $(-\infty, 0]$

$$
|x(t)| \leqslant N e^{-\beta(u-t)}|x(u)| \quad(t \leqslant u),
$$

where $N=e^{3 M h}$ and $\beta=h^{-1} \log 3$. Also it follows from the same proposition that equation (1) has an exponential dichotomy on the interval $[0, \infty)$.

But then Proposition 3 in Coppel [2, p. 70] implies that equation (1) has an exponential dichotomy on $(-\infty, \infty)$ and inequalities (5) and (6), together with the discussion in Coppel [2, p. 11], imply that the constants in the dichotomy can be taken to be $L, \beta$ where $L$ depends only on $M, N, \beta$ and hence only on $M, K$, and $\alpha$.

REMARK. Even in the periodic case this theorem is not trivial. If $A(t)$ is $2 \pi$-periodic we would take $T=2 k \pi$ and suppose that equation (1) has an exponential dichotomy on $[a, a+2 k \pi]$ with constants $K, \alpha$ and projection matrix function $P(t)$. The natural way to proceed would be to show that none of the characteristic multipliers lie on the unit circle. 
We choose $l$ as the least positive integer for which $\lambda=K e^{-2 \pi l \alpha}<1$. Then if $k \geqslant l$ the matrix $M=X(a+2 l \pi) X^{-1}(a)$ has the properties that

$$
|M P| \leqslant \lambda, \quad\left|M^{-1}(I-Q)\right| \leqslant \lambda,
$$

where $P=P(a), Q=P(a+2 l \pi)$. Note also that

$$
M P=Q M .
$$

Now if $Q=P$, we could conclude immediately that the eigenvalues of $M$ had modulus either less than or equal to $\lambda$ or greater than or equal to $\lambda^{-1}$ and we would be finished.

However, in general, $Q \neq P$. We note that equation (1) also has an exponential dichotomy on the interval $[a-2 \pi, a+2(k-1) \pi]$ with constants $K, \alpha$ and projection matrix function $P(t+2 \pi)$ and we may apply Lemma 2 in the following section to deduce that $|P(t+2 \pi)-P(t)|$ is small when $k$ is large. From this it follows that $Q$ is near $P$ and then (7) together with some work imply that the eigenvalues of $M$ do not lie on the unit circle.

One sees from this rough sketch of it that this proof is hardly any simpler than the one used for the general almost periodic case.

3. A refinement of the main result. In this section we prove a refinement of Theorem 1 in which we show that the constants in the dichotomy for equation (1) can be taken close to $K, \alpha$ provided $T$ is sufficiently large and also that the respective projection matrix functions are close to each other. For this, we need a lemma from Palmer [4]. For the reader's convenience, this is quoted here.

Lemma 2. Let $A(t)$ and $B(t)$ be continuous $n \times n$ matrix functions defined on an interval $[a, a+2 T]$ such that

$$
\left|Y(t) Y^{-1}(u)-X(t) X^{-1}(u)\right| \leqslant \delta
$$

for $a \leqslant t, u \leqslant a+2 T$, where $X(t)$ and $Y(t)$ denote fundamental matrices for equations (1) and (2) respectively. Suppose also that equations (1) and (2) have exponential dichotomies on $[a, a+2 T]$ with constants $K, \alpha$ and $L, \beta$ respectively and let $P(t), Q(t)$ be the corresponding projection matrix functions.

Let $\varepsilon>0$ be given with $\varepsilon \leqslant 1 / 3$. Then if $T>0$ and $\delta>0$ satisfy

$$
\frac{1}{4} K^{-2} \varepsilon e^{\alpha \varepsilon T}-K e^{-\alpha \varepsilon T}-L e^{-\beta \varepsilon T} \geqslant \delta, \quad \delta \leqslant \frac{1}{4} \varepsilon e^{-2 \alpha T},
$$

$Q(t)$ and $P(t)$ have the same rank, the inequality $|Q(t)-P(t)|<\varepsilon$ holds for $a+\varepsilon T \leqslant t \leqslant a+(2-\varepsilon) T$, and the constants in the dichotomy for (2) on the interval $[a+\varepsilon T, a+(2-\varepsilon) T]$ can be taken as $K^{2}(1+4 \varepsilon), \alpha$.

With the help of this lemma, we will prove the following theorem.

THEOREM 2. Let $A(t)$ be an $n \times n$ matrix function, defined and almost periodic on $(-\infty, \infty)$, such that equation (1) has an exponential dichotomy on an interval $[a, a+T]$ with constants $K, \alpha$ and projection matrix function $P(t)$.

Let $\varepsilon>0$ be given. Then if $T$ is sufficiently large, depending on $\varepsilon$ and the function $A(t),(1)$ has an exponential dichotomy on $(-\infty, \infty)$ with constants $K^{4}(1+5 \varepsilon), \alpha-\varepsilon$ and the corresponding projection matrix function $Q(t)$ has the same rank as $P(t)$ and 
also the property that if $J$ is any interval of length $(1-\varepsilon) T$, there exists $\tau$ such that when $t \in J, t+\tau \in[a, a+T]$ and

$$
|Q(t)-P(t+\tau)|<\varepsilon
$$

Proof. We assume without loss of generality that $\varepsilon \leqslant 2 / 3$ and then choose $T_{0}$ as the least positive number satisfying

$$
\begin{gathered}
T_{0} \geqslant 2 \alpha^{-1}\left(\sinh ^{-1} 4+\log K\right), \quad T_{0} \geqslant \varepsilon^{-1}(2-\varepsilon)^{-1} \log K^{2}(1+2 \varepsilon), \\
\frac{1}{8} K^{-2} \varepsilon e^{\alpha \varepsilon T_{0} / 2}-K e^{-\alpha \varepsilon T_{0} / 2}-L e^{-\beta \varepsilon T_{0} / 2} \geqslant 1,
\end{gathered}
$$

where $L, \beta$ are the constants in Theorem 1. Then we choose $\delta$ as the largest positive number for which $\delta_{1}=e^{2 M T_{0}}\left(e^{2 \delta T_{0}}-1\right)(M=\sup |A(t)|)$ satisfies

$$
\delta_{1} \leqslant 1, \quad \delta_{1} \leqslant \frac{1}{8} \varepsilon e^{-2 \alpha T_{0}} .
$$

Then we suppose $T \geqslant 2\left(1-\frac{1}{2} \varepsilon\right)^{-1} T_{0}$ and that $T$ is so large that every interval of length $\frac{1}{2} \varepsilon T$ contains a $\delta$-translation number for $A(t)$.

First we see that the hypotheses of Theorem 1 are satisfied and so equation (1) has an exponential dichotomy on $(-\infty, \infty)$ with constants $L, \beta$.

Now let $s$ be an arbitrary real number and let $\tau \in\left[a-s-\frac{1}{4} \varepsilon T, a-s+\frac{1}{4} \varepsilon T\right]$ be a $\delta$-translation number for $A(t)$. Then for all $t$

$$
|A(t+s)-A(t+s+\tau)|<\delta .
$$

Also if $\frac{1}{4} \varepsilon T \leqslant t \leqslant\left(1-\frac{1}{4} \varepsilon\right) T$, we see that $a \leqslant t+s+\tau \leqslant a+T$. Hence the equation

$$
\dot{x}=A(t+s+\tau) x
$$

has an exponential dichotomy on the interval $\left[\frac{1}{4} \varepsilon T,\left(1-\frac{1}{4} \varepsilon\right) T\right]$ with constants $K, \alpha$ and projection matrix function $P(t+s+\tau)$. Also we know that the equation

$$
\dot{x}=A(t+s) x
$$

has an exponential dichotomy on $(-\infty, \infty)$ with constants $L, \beta$ and projection matrix function $Q(t+s)$. Moreover it follows from Lemma 1 and the definition of $\delta_{1}$ that

$$
\left|X(t+s) X^{-1}(u+s)-X(t+s+\tau) X^{-1}(u+s+\tau)\right| \leqslant \delta_{1}
$$

when $t, u \in J$, where $J=\left[c, c+2 T_{0}\right]$ is any interval of length $2 T_{0}$ contained in $\left[\frac{1}{4} \varepsilon T,\left(1-\frac{1}{4} \varepsilon\right) T\right]$.

Then by Lemma 2 with $A(t+s+\tau)$ as $A(t), A(t+s)$ as $B(t)$, and with $\frac{1}{2} \varepsilon$ instead of $\varepsilon$, we deduce that $Q(t)$ and $P(t)$ have the same rank, that

$$
|Q(t+s)-P(t+s+\tau)|<\frac{1}{2} \varepsilon
$$

for $c+\frac{1}{2} \varepsilon T_{0} \leqslant t \leqslant c+\left(2-\frac{1}{2} \varepsilon\right) T_{0}$, and that the constants in the dichotomy of equation (10) on $J$ can be taken as $K^{2}(1+2 \varepsilon)$, $\alpha$.

From the arbitrariness of $J$, it follows that inequality (9) holds for

$$
s+\frac{1}{4} \varepsilon\left(2 T_{0}+T\right) \leqslant t \leqslant s+T-\frac{1}{4} \varepsilon\left(2 T_{0}+T\right) .
$$

Since $T>2 T_{0}$ and $s$ is also arbitrary, we already have the required conclusion for $Q(t)$. 
Now by taking $J=\left[\frac{1}{4} \varepsilon T, \frac{1}{4} \varepsilon T+2 T_{0}\right]$, we see that for any real $s$ the constants in the dichotomy for equation (1) on $\left[s+\frac{1}{4} \varepsilon T+\frac{1}{2} \varepsilon T_{0}, s+\frac{1}{4} \varepsilon T+\left(2-\frac{1}{2} \varepsilon\right) T_{0}\right]$ can be taken as $K^{2}(1+2 \varepsilon), \alpha$. Hence, since $s$ is arbitrary, these can be taken as the constants on any interval of length $(2-\varepsilon) T_{0}$. Then by a standard argument (as, for example in the proof of Theorem 2 in Palmer [4]), we can show that on $(-\infty, \infty)$ the constants can be taken as $\left\{K^{2}(1+2 \varepsilon)\right\}^{2}, \alpha-\left[(2-\varepsilon) T_{0}\right]^{-1} \log K^{2}(1+2 \varepsilon)$ and hence as $K^{4}(1+5 \varepsilon), \alpha-\varepsilon$. Thus the proof of Theorem 2 is complete.

4. Another remark about almost periodic equations. It is well known that if $A(t) \equiv A$, equation (1) has an exponential dichotomy on $(-\infty, \infty)$ if and only if the eigenvalues of $A$ have nonzero real parts. To show that this does not generalize to the almost-periodic case, one can construct counterexamples as follows (the Markus-Yamabe example [3, p. 310] is a special case of this): We take two constant matrices $L$ and $C$ with the properties that $e^{L t}$ is bounded for all $t$, the eigenvalues of $C$ have nonzero real parts but $C-L$ has at least one purely imaginary eigenvalue. Then if we define $A(t)=e^{L t} C e^{-L t}, A(t)$ has constant eigenvalues with nonzero real parts but the kinematic similarity $x=e^{L t} y$ takes equation (1) into the equation

$$
\dot{y}=(C-L) y,
$$

which does not have an exponential dichotomy. So equation (1) cannot have an exponential dichotomy.

\section{REFERENCES}

1. W. A. Coppel, Stability and asymptotic behavior of differential equations, Heath, Boston, Mass., 1965. , Dichotomies in stability theory, Lecture Notes in Math., vol. 629, Springer-Verlag, Berlin and New York, 1978.

3. L. Markus and H. Yamabe, Global stability criteria for differential systems, Osaka Math. J. 12 (1960), 305-317.

4. K. J. Palmer, A perturbation theorem for exponential dichotomies, Proc. Roy. Soc. Edinburgh 103A (1987)

Department of Mathematics and Computer Science, University of Miami, P. O. Box 249085 , Coral Gables, Florida 33124 